\title{
Accuracy and dimensional reproducibility by model scanning, intraoral scanning, and CBCT imaging for digital implant dentistry
}

\author{
Akira Komuro ${ }^{1 *}$, Yoichi Yamada ${ }^{2}$, Satoshi Uesugi ${ }^{1}$, Hiroaki Terashima', Masashi Kimura', Hiroto Kishimoto ${ }^{1}$, \\ Tsutomu lida ${ }^{1}$, Katsuya Sakamoto ${ }^{1}$, Kenichi Okuda ${ }^{1}$, Kaoru Kusano ${ }^{2}$, Shunsuke Baba ${ }^{2}$ and Takashi Sakamoto
}

\begin{abstract}
Background: Recently, it has become possible to analyze implant placement position using the digital matching data of optical impression data of the oral cavity or plaster models with cone beam computed tomography (CBCT) data, and create a highly accurate surgical guide. It has been reported that $C B C T$ measurements were smaller than the actual values, termed shrinkage. Matching of digital data is reliable when the plaster model or intraoral impression values show shrinkage at the same rate as the CBCT data. However, if the shrinkage rate is significantly different, the obtained digital data become unreliable. To clarify digital matching reliability, we examined dimensional reproducibility and shrinkage in measurements obtained with a model scanner, intra-oral scanner (iOS), and $\mathrm{CBCT}$.

Materials and methods: Three implants that were arranged in a triangle were fixed in an acrylic plate. The distance between each implants were measured using model scanner, iOS, and CBCT. The actual size measured by electronic caliper was regarded as control.

Results: All values measured with CBCT were significantly smaller than that of model scanner, iOS, and control $(p<$ 0.001). The model scanner shrinkage was $0.37-0.39 \%$, iOS shrinkage was $0.9-1.4 \%$, and CBCT shrinkage was 1.8-6.9\%. There were statistically significant differences among the shrinkage with iOS, CBCT, and model scanner $(p<0.001)$.

Conclusion: Our findings showed that all measurements obtained with those modalities showed shrinkage as compared to the actual values. In addition, CBCT shrinkage was largest among three different measuring methods. They indicated that data matching between $\mathrm{CBCT}$ and scanner measurements requires attention in regard to the reliability of values obtained with those devices.
\end{abstract}

Keywords: Digital implant dentistry, Surgical guide, Model scanner, Intraoral scanner, CBCT

\section{Introduction}

In recent years, the so called top-down treatments have been widely used to simulate implant placement using findings obtained with cone beam computed tomography $(\mathrm{CBCT})$ by taking into account the design of the superstructure $[1,2]$. Three-dimensional radiographic imaging using CBCT was first introduced in 1998 and

\footnotetext{
* Correspondence: akomuro@komuro-dental.com

'Osaka Academy of Oral Implantology, 1-1-43 Abenosuji, Abenoku, Osaka 545-6008, Japan

Full list of author information is available at the end of the article
}

has become an established diagnostic technique in various dental fields including implant dentistry [3, 4]. Compared with traditional radiographic methods, CBCT offers volumetric data on jaw bones and teeth [5]. Initially, conventional multi-slice computed tomography was used; dental cone beam computed tomography rapidly became more popular due to relatively low radiation doses and reasonable costs [6]. Therefore, CBCT has been widely used as a powerful tool in implant diagnosis and surgical planning. Furthermore, it has become possible to perform accurate implant placement by digital

\section{Springer Open}

(๑) The Author(s). 2021 Open Access This article is licensed under a Creative Commons Attribution 4.0 International License, which permits use, sharing, adaptation, distribution and reproduction in any medium or format, as long as you give appropriate credit to the original author(s) and the source, provide a link to the Creative Commons licence, and indicate if changes were made. The images or other third party material in this article are included in the article's Creative Commons licence, unless indicated otherwise in a credit line to the material. If material is not included in the article's Creative Commons licence and your intended use is not permitted by statutory regulation or exceeds the permitted use, you will need to obtain permission directly from the copyright holder. To view a copy of this licence, visit http://creativecommons.org/licenses/by/4.0/. 
matching of optical impression data of the oral cavity or plaster models with CBCT data. It has become possible to analyze implant placement position using the digital matching data and create a highly accurate surgical guide [6, 7]. However, our previous study showed that CBCT measurements were smaller as compared to the actual values, termed shrinkage [8].

Matching of digital data is reliable when the plaster model or intraoral impression values show shrinkage at the same rate as the CBCT data. However, if the shrinkage rate is significantly different, the obtained digital data become unreliable. Moreover, model scanners are used more frequently than intra-oral scanners (iOS) in clinical practice and have additional factors in regard to dimensional changes, such as expansion with hardening, making it difficult to confirm whether appropriate digital matching has been obtained. Even though there are factors such as shrinkage of the data and dimensional deformation of the resin when making the surgical guide from the matching data, in actual clinical practice, the surgical guide can be set in the mouth with slight adjustments. This is because the degree of shrinkage is not so great as to make it impossible to set, and because of the elasticity of the resin. However, in the severe cases of implant placement, it is important to consider the fact that the data shows shrinkage. In order to clarify the reliability of digital matching, we examined dimensional reproducibility and shrinkage in measurements obtained with model scanner, iOS, and CBCT examinations performed using the same subject model.

\section{Materials and methods}

Three titanium screw type implants (Xive, Dentsply Sirona, USA), each measuring $3.4 \mathrm{~mm}$ in diameter and 11 $\mathrm{mm}$ in length, were fixed in an acrylic plate. They were arranged in a triangle with each side approximately 4-5 $\mathrm{cm}$ in length, and designated as $\alpha, \beta$, and $\gamma$ (Fig. 1). The implants distance was set concerning the average distance between the left and right first molars and between the first molars and adult incisors. For the CBCT examination, a Veraviewepocs 3Df device (Morita, Kyoto, Japan) (voxel size: $125 \mu \mathrm{m}, \mathrm{FoV}: \varnothing 100 \times \mathrm{H} 50 / 80$ ) was used. Cerec Omnicam (Dentsply Sirona, USA) was used for iOS. For the model scanning, the model was constructed of high-strength dental stone (Newfujirock, GC, Tokyo, Japan) after application of a silicone rubber impression material (Dent Silicone Aqua, Shofu, Kyoto, Japan). Then, a Cerec InLabo (Dentsply Sirona, USA) was used for scanning. After CBCT imaging, model scanning and intra-oral scanning, the distances between each implant were automatically visualized by the included software and measured 10 times by 3 dentists with more than 20 years of general clinical experience, with the final values agreed on by consensus. The
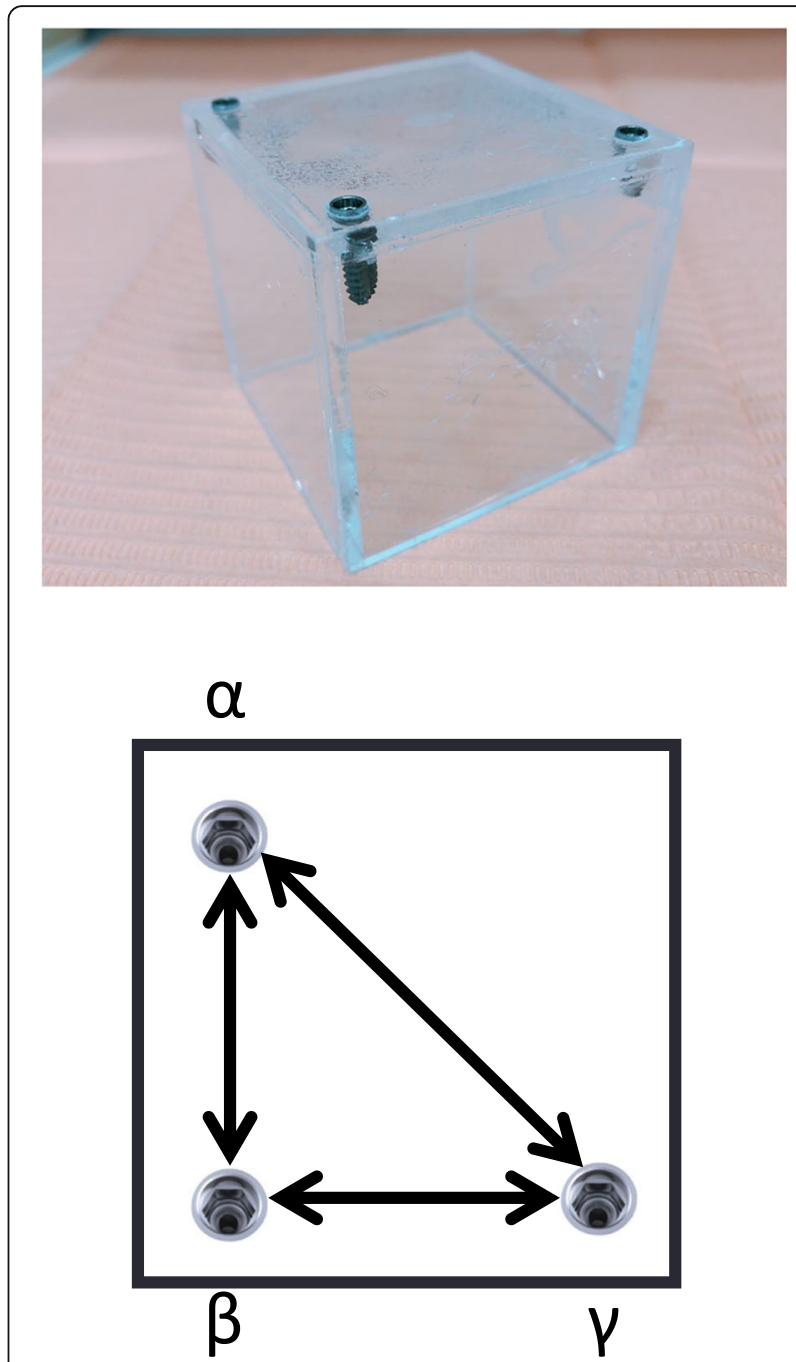

Fig. 1 Model used for scanning. Three titanium screw type implants were fixed to an acrylic plate and arranged in a triangle, with the side lengths ranging from $4-5 \mathrm{~cm}$ and designated as $\alpha, \beta$, and $\gamma$

imaging conditions for CBCT were $90 \mathrm{kV}$ at $5 \mathrm{~mA}$. Actual dimensions between the implants were defined as control. They are determined by measuring 10 times for each implant using an electronic caliper (IP67, HoLEX, Germany).

The statistical analyses were performed using the SigmaPlot software 12.3. Differences among the experimental groups were examined by one-way analysis of variance (ANOVA) tests using Tukey's honest significant difference test. A $p$ value less than 0.05 was considered to be statistically significant.

\section{Results}

The measurements between each implant are shown in Fig. 2. Mean values for the actual measured size (control) and that obtained by the model scanner, iOS, and CBCT for the distance between $\alpha$ and $\beta(\alpha-\beta)$ were 


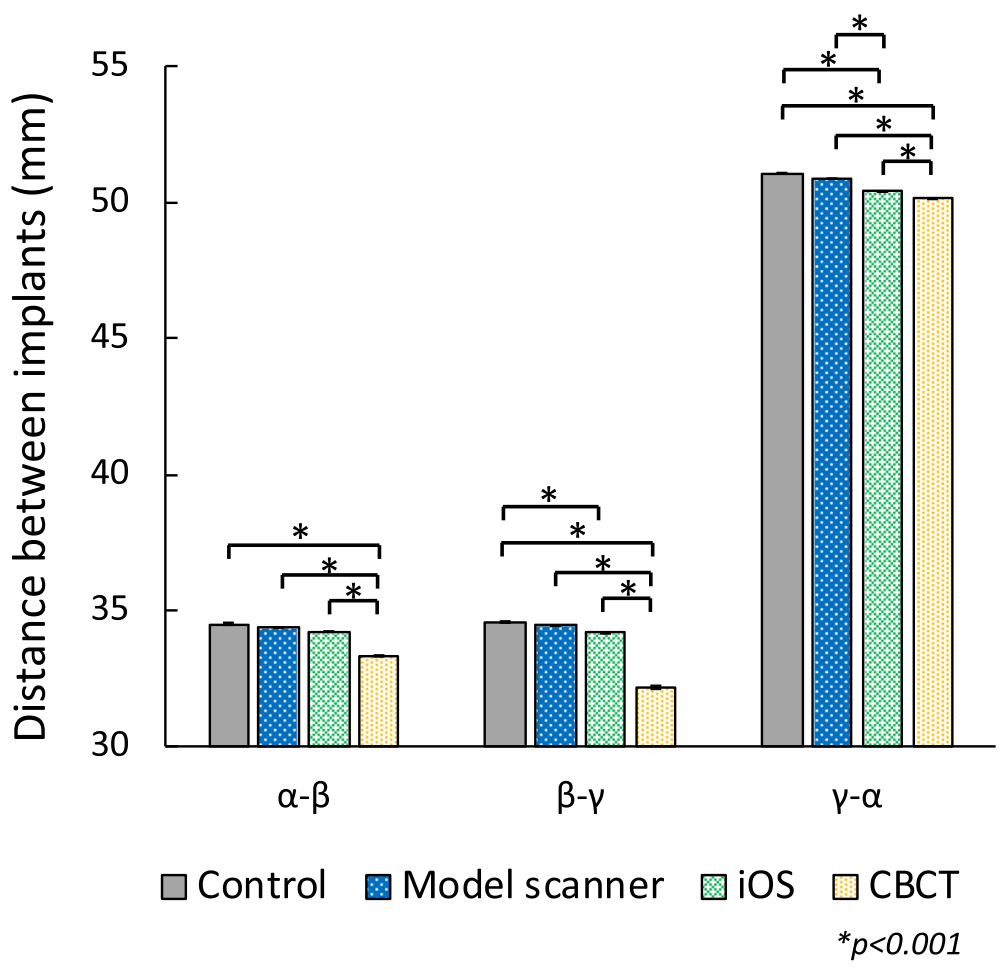

Fig. 2 Distance between each implant. The vertical axis shows the measurements obtained by the model scanner, iOS, and CBCT. Bar: standard deviation

$34.5,34.37,34.22$, and $33.33 \mathrm{~mm}$, respectively, between $\beta$ and $\gamma(\beta-\gamma)$ were $34.57,34.44,34.16$, and $32.17 \mathrm{~mm}$, respectively, and between $\gamma$ and $\alpha(\gamma-\alpha)$ were 51.06, $50.86,50.39$, and $50.13 \mathrm{~mm}$, respectively. The measurements by CBCT were statistically significantly smaller than that of control, model scanner, and iOS at all measurement position $(p<0.001)$. Data obtained by iOS were also significantly smaller than control at $\beta-\gamma$ and $\gamma-\alpha$ $(p<0.001)$. There were no significant differences between measurements by model scanner and control.

In order to clarify the differences among model scanner, $\mathrm{iOS}$, and CBCT, the percentage of measurements by the model scanner, iOS, and CBCT compared to control were analyzed (Fig. 3). CBCT was statistically significantly smaller than both model scanner and iOS at all measurement position $(p<0.001)$. There were also significant differences between model scanner and iOS at $\alpha$ $-\beta$ and $\beta-\gamma(p<0.05)$, and at $\gamma-\alpha(p<0.001)$.

The percentage of shrinkage of the model scanner, iOS, and CBCT was shown in Fig. 4. The average shrinkage rate of the model scanner, $\mathrm{iOS}$, and CBCT for the distance of $\alpha-\beta$ were $0.37 \pm 0.1 \%, 0.81 \pm 0.2 \%$, and $3.41 \pm$ $0.45 \%$, respectively, between $\beta$ and $\gamma$ were $0.37 \pm 0.11 \%$, $1.19 \pm 0.28 \%$, and $6.94 \pm 0.79 \%$, respectively, and between $\gamma$ and $\alpha$ were $0.38 \pm 0.02 \%, 1.37 \pm 0.22 \%, 1.83 \pm 0.26 \%$, respectively. The shrinkage rate of $\mathrm{CBCT}$ was statistically significantly higher than that of model scanner and iOS $(p<0.001)$. There were also significant differences in the shrinkage rate between model scanner and iOS at $\alpha-\beta$ and $\beta-\gamma(p<0.05)$, and at $\gamma-\alpha(p<0.001)$.

\section{Discussion}

In order to clarify the reliability of digital matching, we examined dimensional reproducibility and shrinkage in measurements obtained with model scanner, iOS, and CBCT examinations performed using the same subject model. All values measured with CBCT were significantly smaller than that of model scanner, iOS, and control $(p<0.001)$. The model scanner shrinkage was $0.37-0.39 \%$, iOS shrinkage was $0.9-1.4 \%$, and CBCT shrinkage was $1.8-6.9 \%$.

Since the introduction of digital models in 1990, several ways were developed such as CBCT, iOS, laser scanning, holographic scanning, and stereophotogrammetry [9]. The use of such different types of digital models has increased due to their advantages over plaster models. Especially, CBCT has been used increasingly, because CBCT can obtain more information such as bone levels and root positions [9]. In addition, CBCT is possible to perform virtual setups to simulate results of implant treatment. However, it is still unclear whether the use of software to perform measurements and calculations facilitates and accelerates the process of dental analysis. 


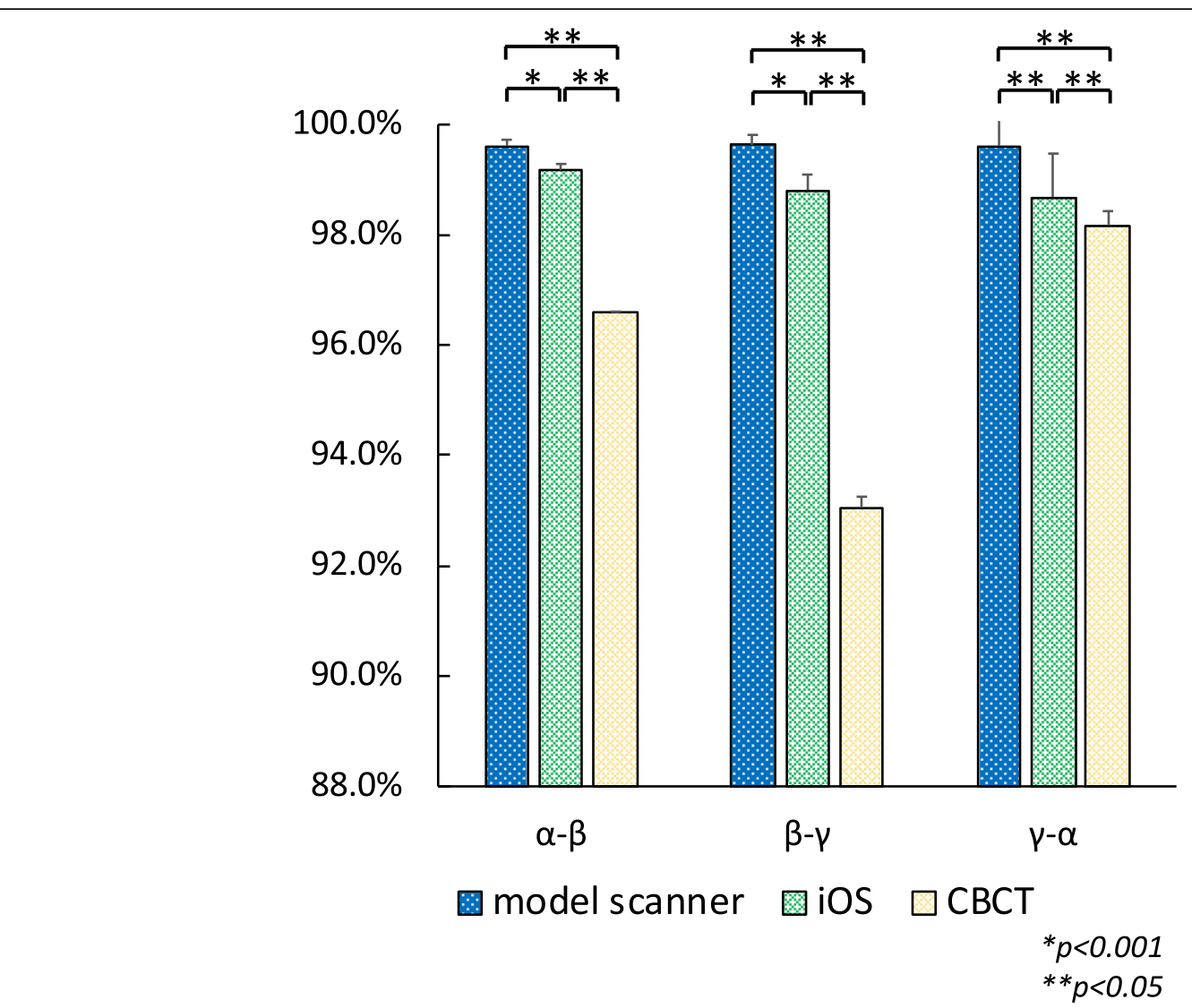

Fig. 3 The percentage of measurements by the model scanner, iOS, and CBCT compared to the control. Bar: standard deviation

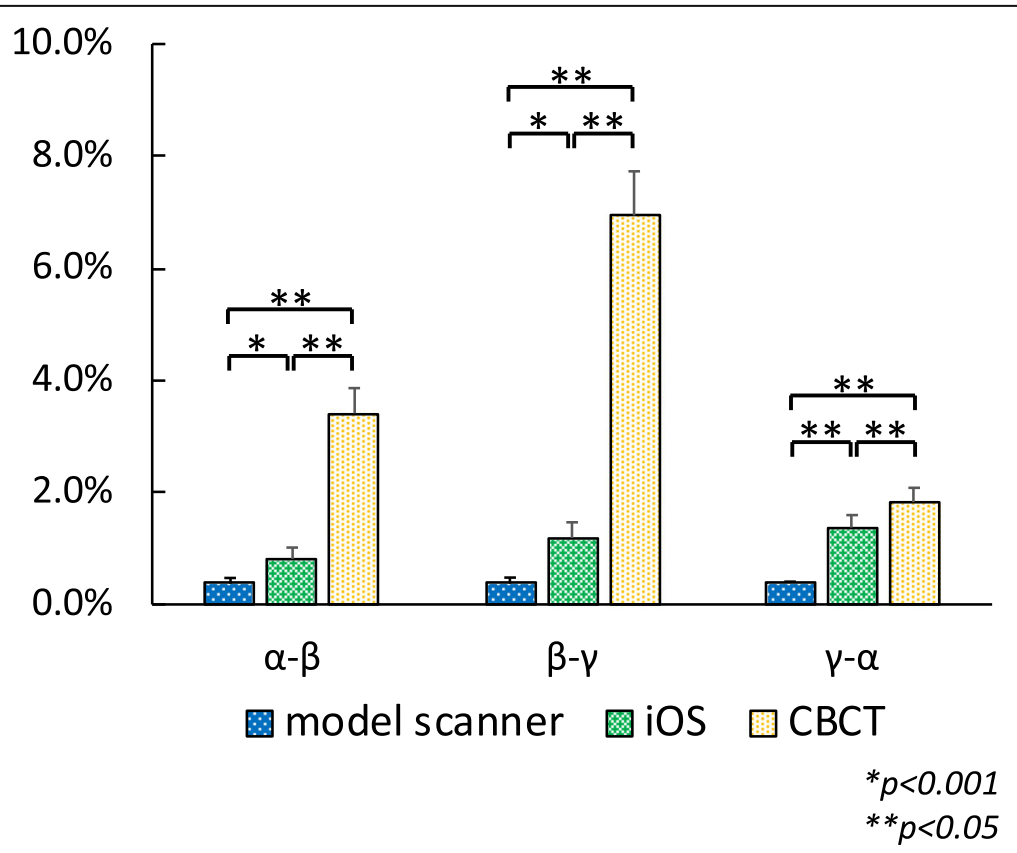

Fig. 4 The shrinkage rate of the model scanner, iOS, and CBCT compared to control. Bar: standard deviation 
Table 1 Overview of shrinkage factors by model scanner, iOS, and CBCT

\begin{tabular}{llll}
\hline & Model scanner & iOS & CBCT \\
\hline System factors & & & CBCT \\
Hardware factors & Camera body & Camera body & Processing software \\
Software factors & CAD software & CAD software & Measurement errors \\
Human factors & Dimensional changes of models & Technical errors during scanning & +++ \\
Amount of shrinkage & + & ++ & + \\
\hline
\end{tabular}

Factors related to shrinkage in measurements obtained by CBCT as compared to the actual values include those related to both hardware and software, as well as human error (Table 1). As for factors related to hardware, it is important to note that the amount of correction is increased because the length of the X-ray tube detector in a panorama multifunction machine is short and the opening angle of the cone beam is wide, while factors related to software are caused by the processes used for edge enhancement and metal artifact reduction. As for human error, that is considered to be primarily related to the individual performing the measurements. In the present study, all measured values were smaller than the actual size because of these factors causing such shrinkage. Factors causing shrinkage of measurements obtained with the model scanners and iOS were also considered to be related to hardware, software, and human error (Table 1). Hardware issues are caused by the performance and scanning mode of the camera body [10], while software issues are related to the CAD software package, and human error is caused by technical errors by the operator during scanning as well as plaster cast production. Shrinkage was smallest with the model scanner, followed by the iOS, and CBCT (Fig. 5). This is thought to be caused that model scanner has larger range for scanning at one time.

In order to consider human and non-human factors separately, hardware and software issues were defined as system factors, then further analysis was conducted. With CBCT, inaccurate measurements caused by human error are very limited, and most of the shrinkage was considered to be caused by system factors. Clinical studies have shown that various imaging objects in the mouth, such as teeth, bones, soft tissues, and metal

\section{Comparison of shrinkage factors}

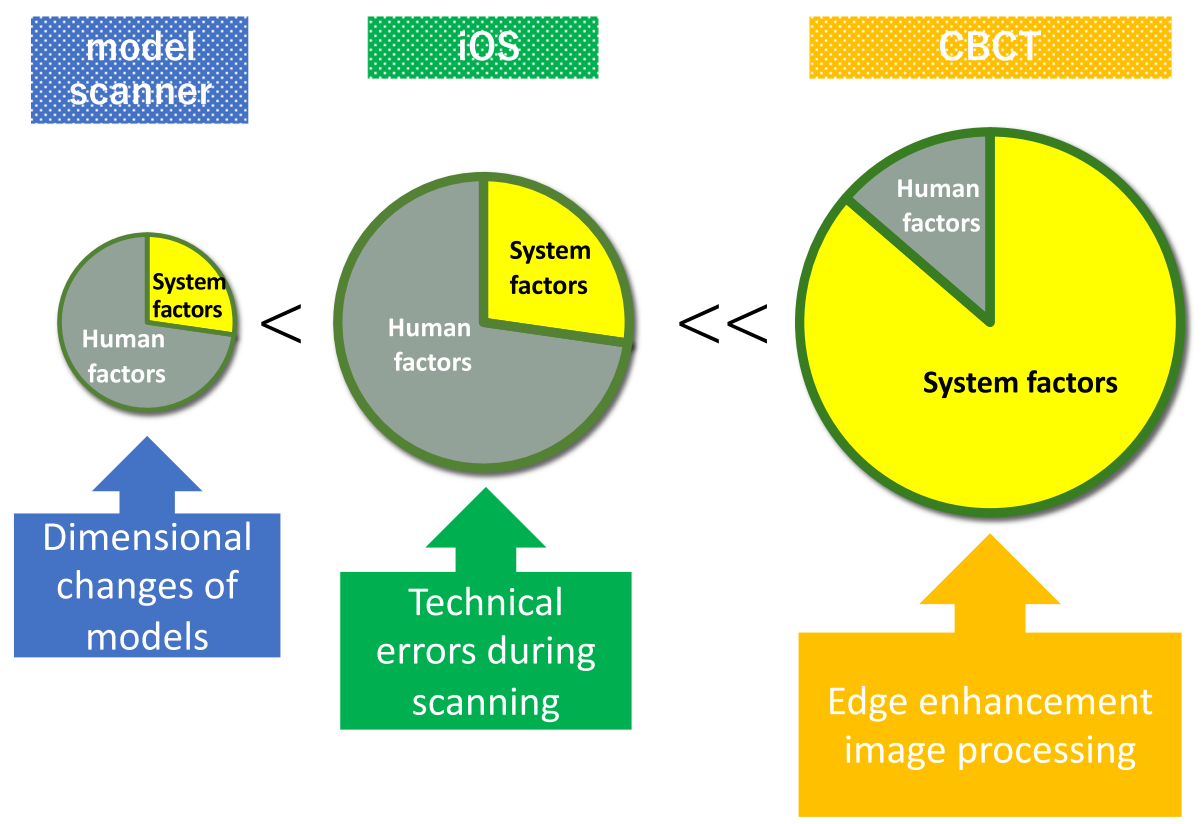

Fig. 5 Factors related to CBCT and scanner measurement shrinkage 
prostheses, have complex effects on imaging and data processing $[11,12]$. The iOS accuracy is also affected by the existence of objects in the mouth, though recent advancements in hardware and software design have been remarkable; thus, it is considered that the influence of human factors is rather large. In other words, the scanning technique of the operator is greatly related to accuracy, including basic techniques such as handling of the camera in cases with a metal prosthesis and moisture condition. With model scanner measurements, though the presence of a metal prosthesis has no influence, dimensional changes of plaster models, such as hardening that causes expansion, are considered to be the major factors leading to errors.

Based on the background information noted above and our findings, the following points are considered important to reduce data matching errors. When an intraoral scanner is employed, mastery of the scanning method is necessary. Furthermore, variations in the ratio of expansion of the plaster model due to hardening can be suppressed by use of an appropriate water ratio for mixing. With CBCT, use of that modality for patients with few metal artifacts caused by metal prostheses is important to reduce system error. Regardless of the method used, the following points are crucial. First, attention must be given to selection of a reference point for data matching. Also, data discrepancies increase with an increased number of implants [13]. Finally, verification of matching accuracy is required for each combination of factors when using $\mathrm{CBCT}$, scanners, and processing software, because CBCT devices use different data contraction modes depending on the manufacturer [14], while model and intra-oral scanners show various levels of accuracy that are dependent on scan mode and recommended scanning method.

Compared to in vitro setups, in a clinical settings, we are likely to be more affected by the "human factors." The difference in the data will be larger in clinical settings and will affect the proper planning and creation of the surgical guide. Therefore, in the severe cases of implant placement, it is important to take into account the fact that the data shows shrinkage.

\section{Conclusion}

In the present study, we determined dimensional reproducibility and shrinkage ratio using model scanners, iOS, and $\mathrm{CBCT}$ with the same subject model to clarify the reliability of digitally obtained measurements. Our findings showed that all measurements obtained with those modalities showed shrinkage as compared to the actual values, with the average and variance greatly different among the 3 methods. In addition, they indicate that data matching between CBCT and scanner measurements, which showed great differences for both average and variance of shrinkage, requires attention in regard to the reliability of values obtained with those devices.

\section{Acknowledgements}

Not applicable

\section{Authors' contributions}

AK participated in the concept/design, data analysis/interpretation, and article writing. YY participated in the data analysis/interpretation and article writing. MK, SB, and TS participated in the concept/design. MK, HK, and KO participated in the measurement of CBCT and data analysis. SU, HT, TI, KS, and KK participated in the data analysis and approval of article. All authors read and approved the final manuscript.

\section{Funding}

The corresponding author funds all materials.

\section{Availability of data and materials}

The datasets used and analyzed during the current study are available from the corresponding author on reasonable request.

\section{Declarations}

Ethics approval and consent to participate

Not applicable

Consent for publication

Consent to publish was obtained from all applicable parties.

\section{Competing interests}

Akira Komuro, Yoichi Yamada, Satoshi Uesugi, Hiroaki Terashima, Masashi Kimura, Hiroto Kishimoto, Tsutomu lida, Katsuya Sakamoto, Kenichi Okuda, Takashi Sakamoto, and Shunsuke Baba state that there are no conflicts of interests.

\section{Author details}

'Osaka Academy of Oral Implantology, 1-1-43 Abenosuji, Abenoku, Osaka 545-6008, Japan. '2Department of Oral Implantology, Osaka Dental University, 1-5-17 Otemae Chuo-ku, Osaka 540-0008, Japan.

Received: 26 January 2021 Accepted: 19 April 2021

Published online: 30 June 2021

\section{References}

1. Oh KC, Jeon C, Park JM, Shim JS. Digital workflow to provide an immediate interim restoration after single-implant placement by using a surgical guide and a matrix-positioning device. J Prosthet Dent. 2019;121(1):17-21. https:// doi.org/10.1016/j.prosdent.2018.03.029.

2. Jansen CE. CBCT technology for diagnosis and treatment planning: what general practitioners should consider. Compend Contin Educ Dent. 2014; 35(10):749-53.

3. Mozzo P, Procacci C, Tacconi A, Tinazzi Martini P, Bergamo Andreis IA. A new volumetric $C T$ machine for dental imaging based on the cone-beam technique: preliminary results. Eur Radiol. 1998;8(9):1558-64. https://doi. org/10.1007/s003300050586.

4. Bornstein MM, Horner K, Jacobs R. Use of cone beam computed tomography in implant dentistry: current concepts, indications and limitations for clinical practice and research. Periodontol 2000. 2017;73(1): 51-72. https://doi.org/10.1111/prd.12161.

5. Jacobs R, Salmon B, Codari M, Hassan B, Bornstein MM. Cone beam computed tomography in implant dentistry: recommendations for clinical use. BMC Oral Health. 2018;18(1):88. https://doi.org/10.1186/s12903-0180523-5.

6. Jacobs R, Quirynen M. Dental cone beam computed tomography: justification for use in planning oral implant placement. Periodontol 2000. 2014;66(1):203-13.

7. Schnutenhaus S, Gröller S, Luthardt RG, Rudolph H. Accuracy of the match between cone beam computed tomography and model scan data in template-guided implant planning: a prospective controlled clinical study. Clin Implant Dent Relat Res. 2018;20(4):541-9. https://doi.org/10.1111/cid.12614. 
8. Kimura M, Kishimoto H, Komuro A, Okuda K, Kubo S, Sakamoto T. Evaluation of the osseointegration using X-ray imaging diagnosis. J Bio-Integ. 2016;6: 47-9.

9. Ferreira JB, Christovam IO, Alencar DS, da Motta AFJ, Mattos CT, CurySaramago A. Accuracy and reproducibility of dental measurements on tomographic digital models: a systematic review and meta-analysis. Dentomaxillofac Radiol. 2017;46(7):20160455. https://doi.org/10.1259/dmfr.2 0160455.

10. Su T, Sun J. Intraoral digital impression technique: a review. J Prosthodont. 2015;24(4):313-21.

11. Abhishek S, Anuj M, Sunita S, Pooja MS, Akhilanand C. Understanding artifacts in cone beam computed tomography. Int J Maxillofacial Imaging. 2016;2(2):51-4

12. Schulze R, Heil U, Groß D, Bruellmann DD, Dranischnikow E, Schwanecke U, et al. Artefacts in CBCT: a review. Dentomaxillofac Radiol. 2011;40(5):265-73. https://doi.org/10.1259/dmfr/30642039.

13. Flügge TV, Att W, Metzger MC, Nelson. Precision of dental implant digitization using intraoral scanners. Int. J. Prosthodont. 2016;29:277-83.

14. Logozzo S, Franceschini G, Kilpela A, Caponi M, Governi L, Blois L. A comparative analysis of intraoral $3 d$ digital scanners for restorative dentistry. Internet J Med Technol. 2011;5:1.

\section{Publisher's Note}

Springer Nature remains neutral with regard to jurisdictional claims in published maps and institutional affiliations.

\section{Submit your manuscript to a SpringerOpen ${ }^{\circ}$ journal and benefit from:}

- Convenient online submission

- Rigorous peer review

- Open access: articles freely available online

High visibility within the field

- Retaining the copyright to your article

Submit your next manuscript at $\boldsymbol{\nabla}$ springeropen.com 